\title{
Evaluation of AGA and Fukuoka Guidelines for EUS and surgical resection of incidental pancreatic cysts
}

\section{(9) $\odot \odot$}

\author{
Authors \\ Alexander Lee ${ }^{1}$, Vivek Kadiyala² ${ }^{2}$ Linda S. Lee ${ }^{3}$ \\ Institutions \\ 1 Texas Digestive Disease Consultants, Dallas, Texas, United \\ States \\ 2 Brigham and Women's Hospital, Division of Gastroenteriology, \\ Hepatology, and Endoscopy, Boston, Massachusetts, United \\ States \\ 3 Brigham and Women's Hospital, Division of Gastroenterology, \\ Hepatology, and Endoscopy, Boston, Massachusetts, United \\ States
}

submitted 17.6.2016

accepted after revision 4.10 .2016

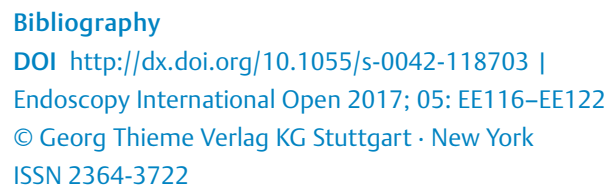

Bibliography

DOI http://dx.doi.org/10.1055/s-0042-118703 |

Endoscopy International Open 2017; 05: EE116-EE122

(c) Georg Thieme Verlag KG Stuttgart · New York

ISSN 2364-3722

\section{Corresponding author}

Linda S. Lee, MD, Brigham and Women's Hospital, Division of Gastroenterology, Hepatology, and Endoscopy, 75 Francis St, Boston, MA, USA 02115

Phone: 617-278-0359

Islee@partners.org

\begin{abstract}
Objectives Management of asymptomatic pancreatic cysts is challenging. Guidelines by the American Gastroenterological Association (AGA) and International Association of Pancreatology (Fukuoka) seek to identify high-risk patients. We assessed performance of these guidelines in selecting patients for endoscopic ultrasound (EUS) and/or surgery.

Methods PART I - We retrospectively studied 143 asymptomatic cysts with magnetic resonance imaging (MRI) followed by EUS. Appropriate selection for EUS was defined as: malignant cytology or surgical pathology, or development of concerning features on MRI as defined by the guidelines. PART II - We retrospectively studied 152 resected cysts to assess the performance of guidelines in selecting cysts for surgery using malignant histology as the outcome. Results PART I - Of 143 EUS, 43 (30.1\%) were male with median age 65.0 years (interquartile range [IQR] 58.0-73.0). AGA guideline demonstrated lower sensitivity $(17.6 \%$ versus $35.3 \%, P=0.03)$, higher specificity $(94.5 \%$ versus $66.1 \%, p<0.001)$, and higher accuracy $(76.2 \%$ versus $58.7 \%, P=0.002)$ than Fukuoka. There was no difference in positive predictive value $(50.0 \%$ versus $24.5 \%, P=$ $0.15)$ and negative predictive value $(78.6 \%$ versus $76.6 \%, \mathrm{p}=0.75)$. PART II - Of 152 resected cysts, 45 (29.8\%) were male with median age 59.0 years (IQR 47.3-66.7). There was no difference in performance characteristics of the guidelines in selecting cysts for surgery. AGA and Fukuoka guidelines missed $25.0 \%$ and $18.8 \%$ of malignant cysts, respectively $(P=1.00)$.

Conclusions For referral to EUS, the AGA guideline was highly specific compared to Fukuoka; both suffered from poor sensitivity, although the Fukuoka guideline was relatively more sensitive than AGA. For referral to surgery, both guidelines have modest sensitivity and specificity and miss a similar percentage of malignant lesions.
\end{abstract}

\section{Introduction}

Incidentally identified pancreatic cysts are an increasingly common problem due to the growing sophistication and increasing use of imaging studies $[1,2]$ The incidence of pancreatic cysts in the United States is estimated to be $3 \%$ to $15 \%$ [3]. Fifteen percent of abdominal magnetic resonance imaging (MRI) studies obtained for non-pancreatic indications incidentally show pancreatic cysts [4]. Different types of cysts have varying risk of progression to high-grade dysplasia (HGD) and/or invasive cancer. Consequently, accurately identifying high-risk cysts is critical to appropriate management. Difficulty arises because currently available diagnostic tools - including computed tomography (CT), MRI, and endoscopic ultrasound (EUS) - have limited ability to identify high-risk lesions [4-7] Moreover, the exact features considered to be high risk and their relative importance is controversial [6, 8-14]
The American Gastroenterological Association (AGA) recently released updated guidelines to direct management of asymptomatic pancreatic cysts [4]. These guidelines differ significantly from the 2012 International Association of Pancreatology Fukuoka guidelines [15]. Notably, the AGA guidelines raise the threshold for EUS and surgery to decrease the number of unnecessary procedures. The evidence on which these new guidelines are based is limited primarily to case series, making the self-reported quality of evidence very low [5]. Further validation of the AGA guidelines is necessary.

In this retrospective study, we assess the performance of the 2015 AGA guidelines and 2012 Fukuoka guidelines in 2 areas that are central to clinical management of asymptomatic pancreatic cysts: 1) criteria for referral to EUS, and 2) criteria for referral to surgery in the ability of the guidelines to identify malignant cysts. 


\section{Methods}

This study was approved by the Institutional Review Board of our institution. Two retrospective databases were utilized in this study.

\section{Part I: Analysis of Guidelines for Referral to EUS}

The first database ("EUS Database”) consisted of all EUS procedures performed at our center from 2001 to 2012 on asymptomatic pancreatic cysts. Inclusion in this study required that the cyst was identified by contrast-enhanced MRI and then evaluated by EUS within 6 months. If surgical resection of the cyst was not performed, the EUS was followed by at least 1 surveillance magnetic resonance image (MRI) within the subsequent 12 months. The following data were collected: demographic information (gender, age), cyst features on MRI (cyst size, number of cysts, location, mural nodule, thick wall, maximum pancreatic duct (PD) size, lymphadenopathy), cyst features on EUS (size, multiplicity, location, mural nodule, thick wall, maximum PD size), cyst fluid analysis (cytology, amylase, CEA, DNA mutation analysis), surgical features (type of surgery, surgical pathology). The criteria for referral to EUS were evaluated for the 2015 AGA and 2012 Fukuoka guidelines. The 2015 AGA guidelines stipulated that MRI should demonstrate at least 2 high-risk features (cyst size $\geq 30 \mathrm{~mm}$, main PD $>3 \mathrm{~mm}$, mural nodule or solid component) while the 2012 Fukuoka guidelines required at least 1 worrisome feature on MRI (cyst size $\geq 30 \mathrm{~mm}$, enhanced thickened cyst walls, non-enhanced mural nodules, main PD 5-9 mm, abrupt change in main PD caliber with distal pancreatic atrophy, lymphadenopathy) $[4,15]$.

For each set of guidelines, cysts were separated into 2 groups - those that did versus those that did not meet criteria for EUS referral - and endpoints were compared between the groups. Primary endpoints included the following: A) positive fine-needle aspiration (FNA) cytology for malignancy; B) positive surgical pathology for malignancy, defined as carcinoma; or $C$ ) if neither $A$ nor $B$, then subsequent MRI (within 12 months after EUS) with concerning features, defined as development of high-risk or worrisome features according to the respective guidelines. This short-term follow-up with imaging was chosen because our goal was to assess whether performing EUS according to each set of guidelines appropriately identified highrisk cysts at the time of EUS. Sensitivity, specificity, positive predictive value, negative predictive value, and accuracy of each set of guidelines were calculated for the endpoints.

\section{Part II: Analysis of Guidelines for Referral to Surgical Resection}

The second database ("Pathology Database") consisted of all surgically resected asymptomatic pancreatic cysts at our center from 1995 to 2013. Inclusion in this study required that all cysts had undergone contrast-enhanced MRI and/or EUS preoperatively. The same data were collected as for the EUS database. The criteria for surgical referral were evaluated for the 2015 AGA and 2012 Fukuoka guidelines. The 2015 AGA guidelines mandated 1 of the following before surgery: 1) malignant cytology on EUS-FNA; or 2) at least 2 high-risk features on MRI and/or EUS (cyst size $\geq 30 \mathrm{~mm}$, main PD $>3 \mathrm{~mm}$, mural nodule or solid component) [4]. The 2012 Fukuoka guidelines required 1 of the following: 1) MRI with any high-risk stigmata (obstructive jaundice in a patient with cystic lesion in the head of the pancreas, enhancing solid component within cyst, main PD $\geq 10 \mathrm{~mm}$ ); or 2) EUS with any concerning features (mural nodule, main PD with thickened walls/intraductal mucin/nodule, cytology suspicious or positive for malignancy) [15]. For each set of guidelines, cysts were separated into 2 groups-those that did versus those that did not meet criteria for referral for surgical resection - and endpoints were compared between the groups. The primary endpoint was surgical pathology demonstrating malignancy, defined as carcinoma. Sensitivity, specificity, positive predictive value, negative predictive value, and accuracy of each set of guidelines were calculated for the endpoint.

\section{Statistical analysis}

Sensitivity, specificity, predictive values, overall accuracy, and referral rates were calculated for the AGA and Fukuoka guidelines. Sensitivity, specificity, and overall accuracy were compared using McNemar's test for paired data. Medians and interquartile ranges (IQR) were reported. Statistical significance was defined as $P<0.05$ (SAS, Cary, NC).

\section{Results}

\section{Part I: Analysis of guidelines for referral to EUS}

A total of 143 EUS examinations with antecedent MRI were identified. Of them, 43 (30.1\%) were done on males with median age of 65.0 years (IQR $58.0-73.0$ ). The median cyst size was $18.0 \mathrm{~mm}$ (IQR $11.0-26.0$ ) with $63.6 \%$ in the head, $21.7 \%$ in the body, and $14.7 \%$ in the tail. MRI identified $25.9 \%$ with size $\geq 3 \mathrm{~cm}, 14.0 \%$ with dilated main PD, and $6.3 \%$ with solid component. By subsequent EUS, $20.3 \%$ had size $\geq 3 \mathrm{~cm}, 16.1 \%$ had dilated main PD, and $5.6 \%$ had a solid component. FNA was performed in 105 (73.4\%) EUS exams; 4 (2.8\%) cysts had malignant cytology. Aspiration was not performed in 38 cysts due to the following reasons: 19 cysts $<1 \mathrm{~cm}, 11$ cysts for technical reasons, and 8 cysts because the results would not alter management. Surgery was performed on 23 (16.1\%) cysts; 5 of these were malignant, 2 additional cysts had high-grade dysplasia, and the remaining 16 included 6 branch duct intraductal papillary mucinous neoplasm, 2 mixed intraductal papillary mucinous neoplasm, 1 mucinous cystic neoplasm, 3 neuroendocrine tumor, 2 pseudocyst, 1 chronic pancreatitis, and 1 normal pancreas.

The overall performances of both the AGA and Fukuoka guidelines for identifying high-risk lesions are shown in $>$ Table 1. The AGA guidelines had significantly lower sensitivity (17.6\%) compared to the Fukuoka guidelines ( $35.3 \%, P=0.03$ ). However, the AGA guidelines had significantly higher specificity (94.5\%) compared to the Fukuoka guidelines $(66.1 \%, P<0.01)$. The AGA guidelines did demonstrate overall greater accuracy compared to the Fukuoka guidelines $(76.2 \%$ versus $58.7 \%, P<0.01)$. When adjusted for selection of cysts with HGD in addition to carcinoma, results were similar; the AGA and Fukuoka guide- 
Table 1 Guideline performance for referral to EUS.

\begin{tabular}{|l|c|c|r|}
\hline & AGA 2015 & Fukuoka 2012 & P value \\
\hline Sensitivity & $17.6 \%$ & $35.3 \%$ & 0.031 \\
\hline Specificity & $94.5 \%$ & $66.1 \%$ & $<0.001$ \\
\hline $\begin{array}{l}\text { Positive Predictive } \\
\text { Value }\end{array}$ & $50.0 \%$ & $24.5 \%$ & 0.154 \\
\hline $\begin{array}{l}\text { Negative Predictive } \\
\text { Value }\end{array}$ & $78.6 \%$ & $76.6 \%$ & 0.747 \\
\hline \begin{tabular}{l} 
Accuracy \\
\hline
\end{tabular} & $76.2 \%$ & $58.7 \%$ & $<0.001$ \\
\hline
\end{tabular}

lines had sensitivities of $16.7 \%$ and $33.3 \%$, respectively $(P=$ 0.03 ), and specificities of $94.4 \%$ and $65.4 \%$, respectively $(P<$ $0.01)$.

By the AGA guidelines, only $8.4 \%$ of patients would have been referred for EUS compared to $34.3 \%$ by the Fukuoka guidelines $(P<0.01)$. $>$ Fig. 1 and $>$ Fig. 2 demonstrate the application of the AGA and Fukuoka guidelines to the EUS Database, respectively, for referral or non-referral to EUS, along with subsequent surgical or surveillance endpoints; patients who underwent EUS and/or surgery despite not meeting guideline criteria are also shown. - Table 2 shows the 7 pancreatic cysts with malignant histology and with high grade dysplasia from the EUS Database, with subsequent confirmation by operative resection; all 3 FNA with malignant cytology underwent resection which confirmed malignancy in all of those cases. According to the AGA guidelines, 3 of the 5 (60\%) resected malignant cysts did not meet criteria for EUS referral; in comparison, by the Fukuoka guidelines, 1 of the 5 (20\%) resected malignant cysts did not meet EUS referral criteria. Of note, 1 of 5 (20\%) malignant cysts had imaging findings on MRI which did not meet sufficient criteria for EUS referral according to either the AGA or Fukuoka guidelines. When adjusted for selection of cysts with HGD in addition to carcinoma, results were similar. By AGA guidelines, 5 of the 7 (71.4\%) resected malignant cysts did not meet criteria for EUS referral, whereas by the Fukuoka guidelines, 3 of the 7 (42.9\%) resected malignant cysts did not meet EUS referral criteria.

\section{Part II: Analysis of guidelines for referral to surgical resection}

There were 152 surgically resected asymptomatic pancreatic cysts included in this analysis ( $>$ Table 3 ). Of them, 45 (29.8\%) were male with median age 59.0 years (IQR 47.3-66.7). Median cyst size was $33.0 \mathrm{~mm}$ (IQR 20.0-42.0) with $33.8 \%$ in the head, $30.5 \%$ in the body, and $35.7 \%$ in the tail. Cyst size was $\geq 3 \mathrm{~cm}$ in $69.5 \%$, with dilated main PD in $26.5 \%$ and solid component in $18.5 \%$. There were 16 malignant cases (10.5\%).

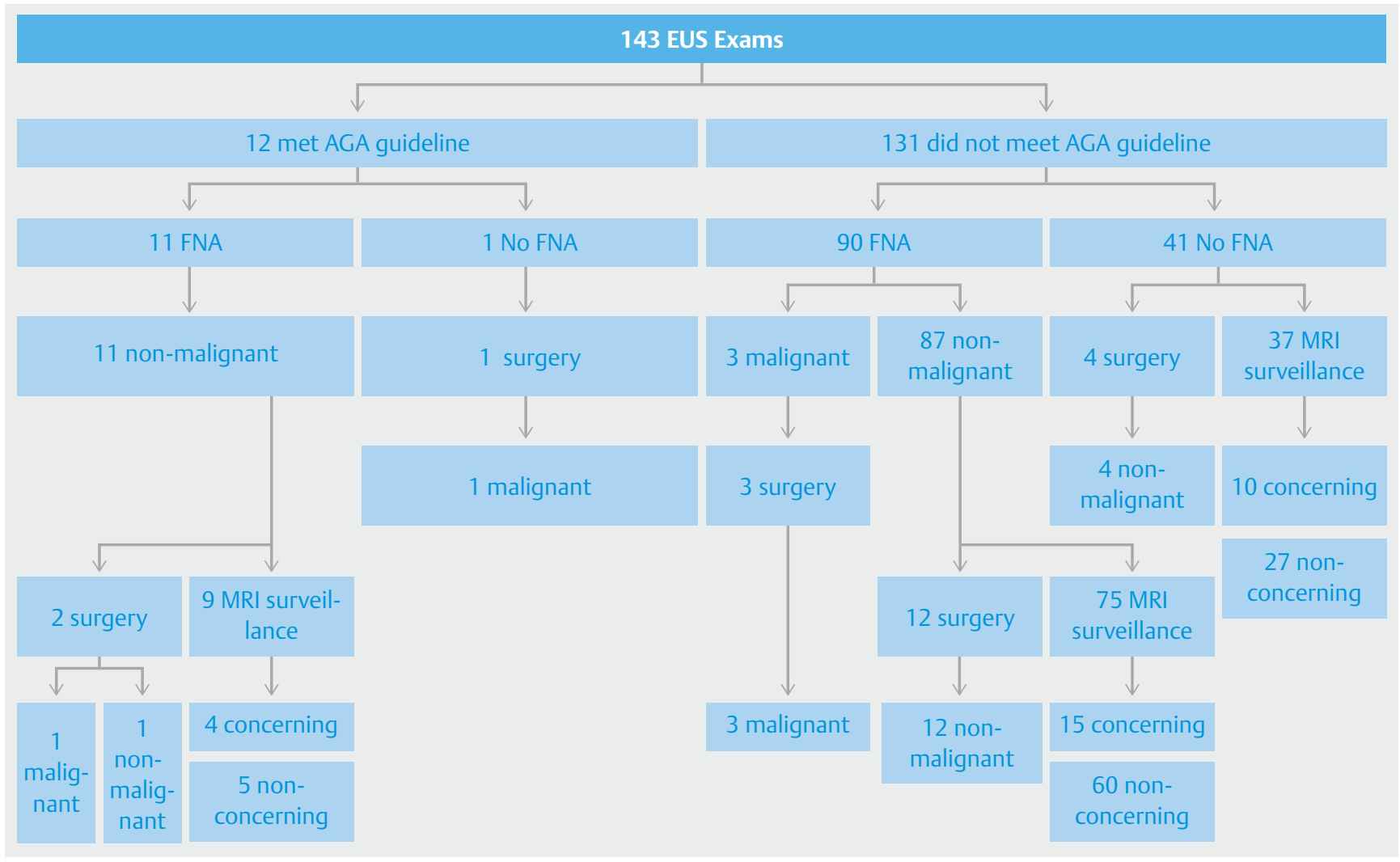

- Fig. 1 Flowchart of application of AGA Guideline for referral for EUS. For MRI surveillance, "concerning" indicates identification of additional high-risk features; "non-concerning" specifies unchanged or smaller cysts with no additional high-risk features. EUS, endoscopic ultrasound; FNA, fine-needle aspiration; MRI, magnetic resonance imaging. 


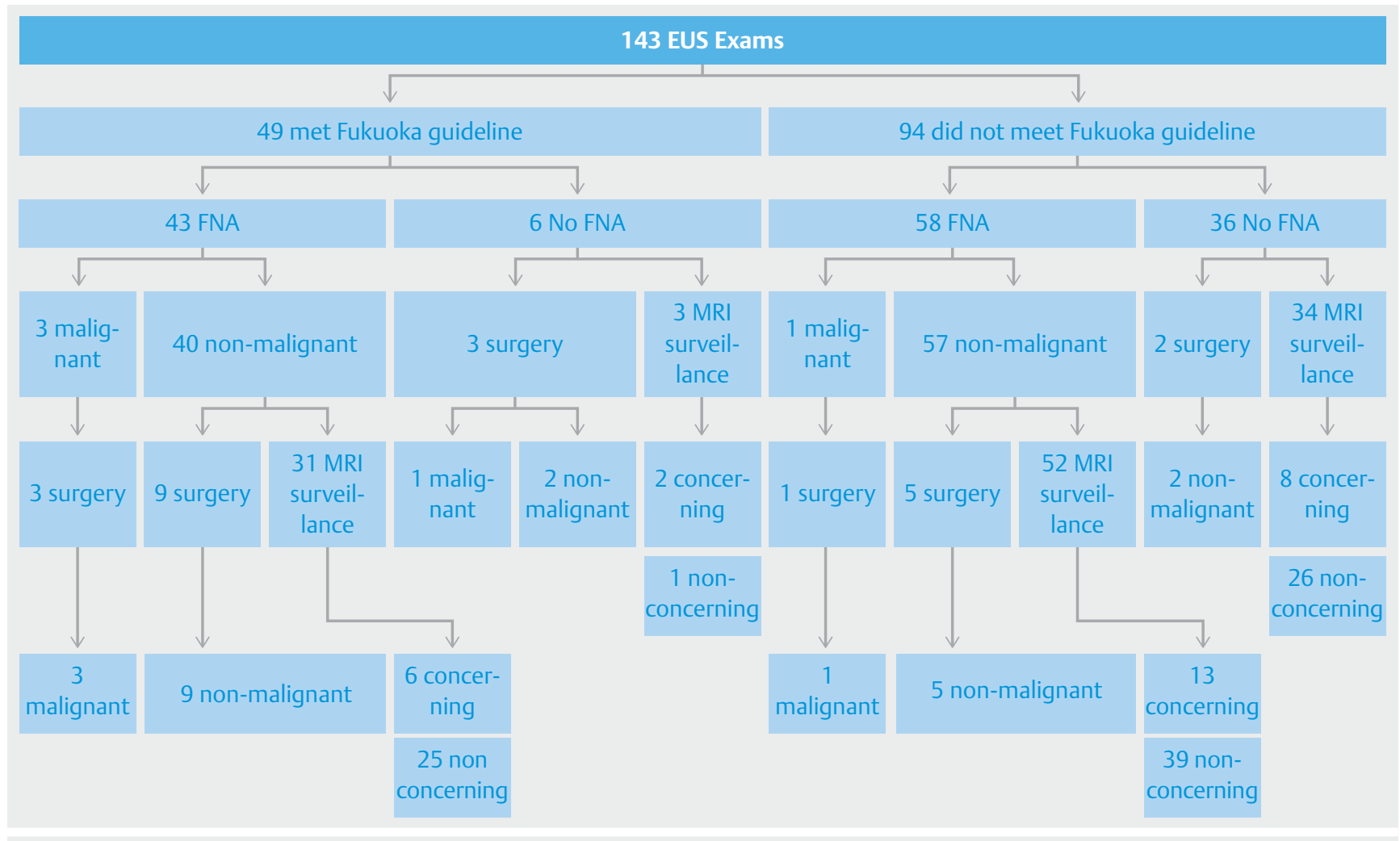

- Fig. 2 Flowchart of application of Fukuoka Guideline for referral for EUS. For MRI surveillance, "concerning" indicates identification of additional high-risk features; "non-concerning" specifies unchanged or smaller cysts with no additional high-risk features. EUS, endoscopic ultrasound; FNA, fine-needle aspiration; MRI, magnetic resonance imaging.

By the AGA guidelines, $30.9 \%$ of patients would have been referred for surgery compared to $36.2 \%$ by the Fukuoka guidelines $(P=0.40)$. The overall performances of both the AGA and Fukuoka guidelines are shown in $>$ Table 4 . The 2 guidelines did not differ significantly with respect to any of the performance indices including sensitivity, specificity, positive predictive value, negative predictive value, and accuracy. Fig. 3 and - Fig. 4 demonstrate the application of the AGA and Fukuoka guidelines to the Pathology Database, respectively, for referral or non-referral to surgical resection along with subsequent surgical endpoints. - Table 5 shows the 16 pancreatic cysts in the Pathology Database with malignant histology at operative resection. According to the AGA guidelines, 4 of the 16 (25\%) malignant cysts did not meet criteria for surgical referral; according to the Fukuoka guidelines, 3 of the 16 (18.8\%) malignant cysts did not meet surgical referral criteria $(P=1.00)$. Of note, 2 of $16(12.5 \%)$ had imaging findings on MRI or EUS which did not meet sufficient criteria for surgical referral according to either the AGA or Fukuoka guidelines. When adjusted for selection of cysts with HGD in addition to carcinoma, results were similar; by AGA guidelines, 5 of the 17 (29.4\%) malignant cysts did not meet criteria for surgical referral, whereas by the Fukuoka guidelines, 3 of the $17(17.6 \%)$ malignant cysts did not meet surgical referral criteria $(P=0.69)$.

If the AGA guidelines had been strictly applied, 101 of 136 (74.3\%) of surgeries on non-malignant cysts would have been prevented, whereas adherence to the Fukuoka guidelines would have prevented 94 of 136 (69.1\%) surgeries on non-malignant cysts $(P=0.42)$.

\section{Discussion}

We evaluated both the 2012 Fukuoka and 2015 AGA guidelines for asymptomatic pancreatic cysts in their recommendations for referring patients to EUS and surgery by using malignant lesions as the outcome. The AGA guidelines were more accurate than the Fukuoka guidelines in recommending patients for EUS, due to markedly higher specificity. Both suffered from very low sensitivity, although the Fukuoka guidelines were relatively more sensitive than the AGA guidelines. Given that the AGA guidelines had higher specificity but lower sensitivity in referring patients for EUS, if the goal is only to send patients to EUS who have a high-risk lesion, the AGA guidelines meet this goal better than the Fukuoka guidelines. However, this is at the expense of missing $71.4 \%$ of malignant cysts in our study. Furthermore, EUS-FNA with cyst fluid analysis is not only important for identifying malignant cysts, but it also adds value by differentiating neoplastic from non-neoplastic cysts [6]. The possibility of improved diagnosis by incorporating molecular markers into traditional cyst fluid analysis increases the potential value of EUS-FNA even further [16].

In evaluating the criteria for surgical referral, both the AGA and Fukuoka guidelines performed similarly with modest sensitivity and specificity. While the specificity of the AGA guidelines 


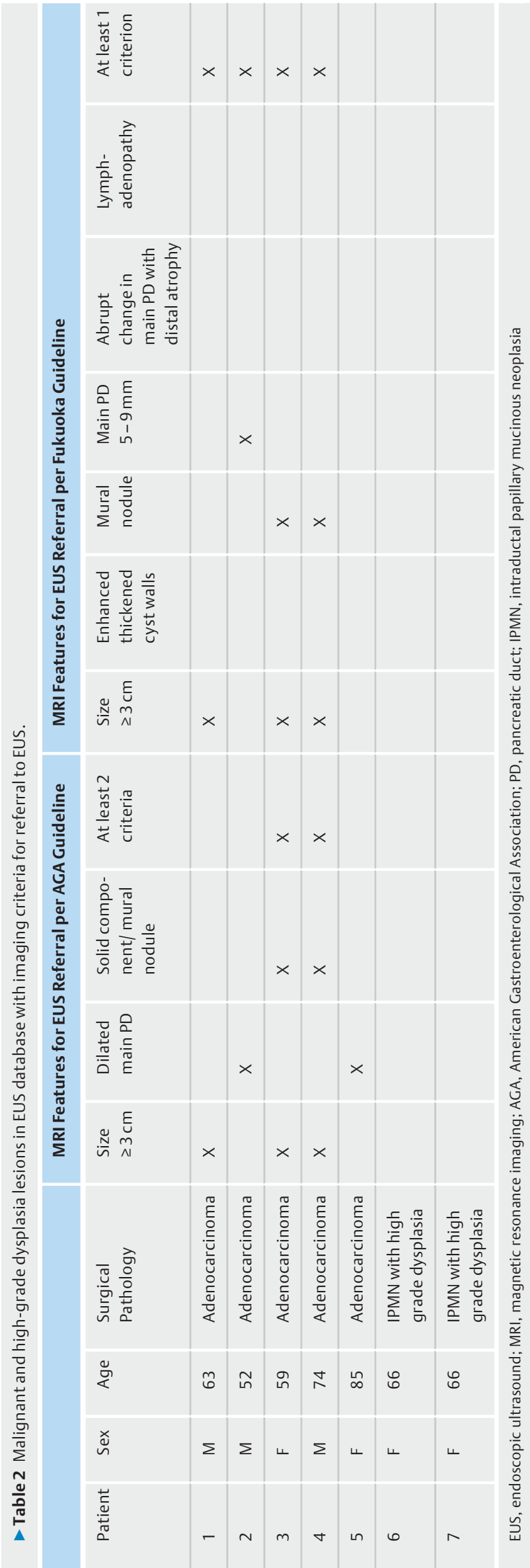

- Table 3 Histology of surgically resected cysts.

\begin{tabular}{|l|r|r|}
\hline Cyst Type & Number & Percentage \\
\hline Adenocarcinoma NOS & 4 & $2.6 \%$ \\
\hline Autoimmune pancreatitis & 1 & $0.7 \%$ \\
\hline BD IPMN & 36 & $23.7 \%$ \\
\hline BD IPMN with adenocarcinoma & 8 & $5.3 \%$ \\
\hline Chronic pancreatitis & 2 & $1.3 \%$ \\
\hline Lymphoepithelial cyst & 2 & $1.3 \%$ \\
\hline MD IPMN & 3 & $2.0 \%$ \\
\hline Mixed IPMN & 5 & $3.3 \%$ \\
\hline Mucinous cystic neoplasm & 24 & $15.7 \%$ \\
\hline Mucinous cystic neoplasm with & 2 & $1.3 \%$ \\
\hline adenocarcinoma & & \\
\hline Neuroendocrine tumor & 8 & $5.3 \%$ \\
\hline Normal pancreas & 1 & $0.7 \%$ \\
\hline Poorly differentiated carcinoma & 1 & $0.7 \%$ \\
\hline Pseudocyst & 4 & $2.6 \%$ \\
\hline Serous cystadenoma & 35 & $23.0 \%$ \\
\hline Simple cyst & 2 & $1.3 \%$ \\
\hline Solid pseudopapillary neoplasm & 14 & $9.2 \%$ \\
\hline TOTAL & 152 & $100 \%$ \\
\hline OOS, & & \\
\hline
\end{tabular}

NOS, not otherwise specified; BD, branch duct; MD, main duct; IPMN, intraductal papillary mucinous neoplasm

D Table4 Guideline performance for referral to surgery.

\begin{tabular}{|l|c|c|c|}
\hline & AGA 2015 & Fukuoka 2012 & P value \\
\hline Sensitivity & $75.0 \%$ & $81.3 \%$ & 1.000 \\
\hline Specificity & $74.3 \%$ & $69.1 \%$ & 0.144 \\
\hline $\begin{array}{l}\text { Positive predictive } \\
\text { value }\end{array}$ & $25.5 \%$ & $23.6 \%$ & 1.000 \\
\hline $\begin{array}{l}\text { Negative predictive } \\
\text { value }\end{array}$ & $96.2 \%$ & $96.9 \%$ & 1.000 \\
\hline \begin{tabular}{l} 
Accuracy \\
\hline
\end{tabular} & $74.3 \%$ & $70.4 \%$ & 0.115 \\
\hline
\end{tabular}

trended higher, this was not significantly different from the Fukuoka guidelines. The AGA guidelines missed $29.4 \%$ of malignancies while the Fukuoka guidelines failed to identify $17.6 \%$ of malignant cysts. Furthermore, $74.5 \%$ of pancreatic cysts referred for surgery by the AGA or the Fukuoka guidelines were unnecessary. Even with using both the AGA and Fukuoka guidelines, $11.8 \%$ of malignant cysts were not identified. Therefore, the goal of decreasing unnecessary high-risk pancreatic surgeries while only sending those patients with high-risk lesions for surgery remains unfulfilled by both sets of guidelines. We believe that when faced with an incidental pancreatic cyst, all pa- 


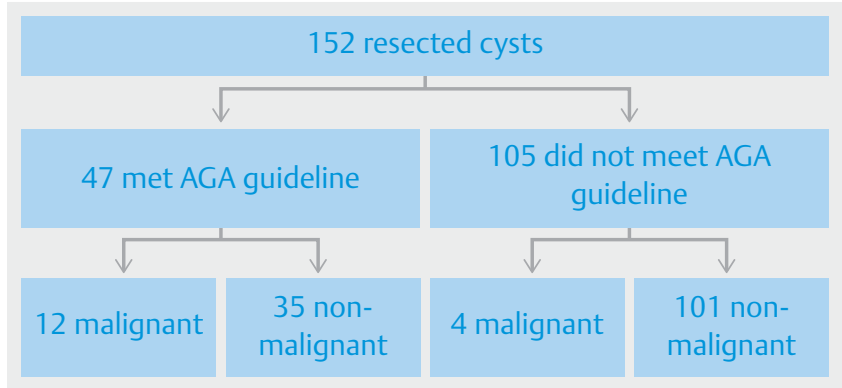

- Fig. 3 Flowchart of application of AGA Guideline for referral to surgery.

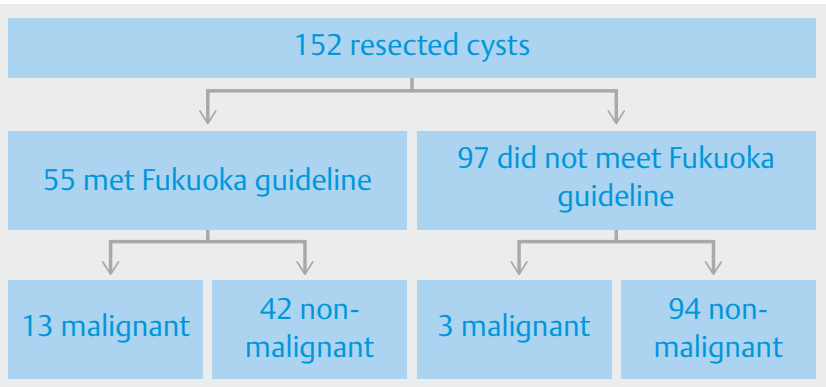

Fig. 4 Flowchart of application of Fukuoka Guideline for referral to surgery.

- Table 5 Malignant lesions in pathology database with criteria for surgical referral.

\begin{tabular}{|c|c|c|c|c|c|c|}
\hline Patient & Sex & Age & $\begin{array}{l}\text { AGA Guideline } \\
\text { for Surgical } \\
\text { Referral }\end{array}$ & $\begin{array}{l}\text { Fukuoka Guide- } \\
\text { line for Surgical } \\
\text { Referral }\end{array}$ & EUS-FNA Cytology & Surgical Pathology \\
\hline 1 & $\mathrm{~F}$ & 51 & $x$ & $x$ & $\mathrm{n} / \mathrm{a}$ & $\begin{array}{l}\text { Mucinous cystadenoma with } \\
\text { adenocarcinoma }\end{array}$ \\
\hline 2 & M & 60 & $x$ & $x$ & Malignant & BD-IPMN with adenocarcinoma \\
\hline 3 & $\mathrm{~F}$ & 60 & & $x$ & $\mathrm{n} / \mathrm{a}$ & BD-IPMN with adenocarcinoma \\
\hline 4 & M & 76 & $x$ & & Atypical & BD-IPMN with adenocarcinoma \\
\hline 5 & $\mathrm{~F}$ & 63 & $x$ & $x$ & Malignant & BD-IPMN with adenocarcinoma \\
\hline 6 & $\mathrm{~F}$ & 77 & & & Atypical & BD-IPMN with adenocarcinoma \\
\hline 7 & $\mathrm{~F}$ & 63 & $x$ & $x$ & Malignant & BD-IPMN with adenocarcinoma \\
\hline 8 & M & 81 & $x$ & $x$ & Malignant & BD-IPMN with adenocarcinoma \\
\hline 9 & $\mathrm{~F}$ & 80 & & $x$ & $\mathrm{n} / \mathrm{a}$ & BD-IPMN with adenocarcinoma \\
\hline 10 & M & 54 & $x$ & $x$ & $\begin{array}{l}\text { Neoplastic cells consistent } \\
\text { with neuroendocrine tumor }\end{array}$ & Pancreatic endocrine carcinoma \\
\hline 11 & M & 63 & $x$ & $x$ & Malignant & $\begin{array}{l}\text { Poorly differentiated carcinoma } \\
\text { with cystic degeneration }\end{array}$ \\
\hline 12 & M & 52 & $x$ & $x$ & Malignant & BD-IPMN with adenocarcinoma \\
\hline 13 & $\mathrm{~F}$ & 77 & $x$ & $x$ & Malignant & BD-IPMN with adenocarcinoma \\
\hline 14 & $\mathrm{~F}$ & 59 & $x$ & $x$ & $\mathrm{n} / \mathrm{a}$ & MCN with adenocarcinoma \\
\hline 15 & M & 74 & $x$ & $x$ & Benign & $\begin{array}{l}\text { Adenocarcinoma with cystic } \\
\text { degeneration }\end{array}$ \\
\hline 16 & $\mathrm{~F}$ & 75 & & & Benign & BD-IPMN with adenocarcinoma \\
\hline
\end{tabular}

BD-IPMN, branch duct intraductal papillary mucinous neoplasm; HCD, high-grade dysplasia; MCN, mucinous cystic neoplasm

tients should undergo a good-quality MRI. If the lesion is at least $2 \mathrm{~cm}$, has at least 1 high-risk feature, or an MRI is not possible, an EUS should be performed to identify mucinous as well as malignant cysts.

Performance of the Fukuoka guidelines is similar to a recent paper of surgically resected pancreatic cysts [8]. Our application of all incidental pancreatic cysts to the Fukuoka guidelines rather than limiting to mucinous cysts may have underestimated its performance as suggested by a previous study that eval- uated the original Sendai guidelines for all resected pancreatic cysts $[17,18]$. However, as the AGA guidelines have done, it is more practical to develop guidelines that apply to all cystic lesions, which is why we applied the Fukuoka guidelines to all asymptomatic pancreatic cysts.

Our paper has certain limitations, which include the retrospective, single-center nature of the study. Ideally all patients who have undergone MRI followed by EUS and/or surgery would have been studied to evaluate the entire sequence of 
the AGA guidelines in all these patients. However, our patient population was limited to those patients who had undergone EUS and/or surgical resection, which may have biased the study towards selecting out more high-risk patients. Therefore, if the AGA guidelines were applied to all incidental pancreatic cysts identified on MRI imaging at our institution, its performance would likely be inferior to that described in our paper. Furthermore, similar suboptimal performance of the AGA guidelines was demonstrated in a recently published retrospective series containing 41 patients with pancreatic cysts with available diagnostic pathology. In that study, the AGA guidelines were shown to have $62 \%$ sensitivity, $79 \%$ specificity, $57 \%$ positive predictive value, and $82 \%$ negative predictive value for advanced neoplasia; also, $45 \%$ of intraductal papillary mucinous neoplasms with adenocarcinoma or high-grade dysplasia were missed [19].

\section{Conclusion}

In conclusion, for referral to EUS, the AGA guidelines were highly specific compared to Fukuoka; both suffer from poor sensitivity, although the Fukuoka guidelines were relatively more sensitive than AGA. Regarding surgical referral, both sets of guidelines perform with only modest sensitivity and specificity in identifying high-risk pancreatic cystic lesions. Therefore, more accurate diagnostic studies are necessary to better identify lesions needing further evaluation and surgical resection in order to optimize resource utilization and decrease risk to the patient from both unnecessary procedures as well as delay of care.

\section{Competing interests}

None

References

[1] Laffan TA, Horton KM, Klein AP et al. Prevalence of unsuspected pancreatic cysts on MDCT. AJR Am J Roentgenol 2008; 191: 802-807

[2] de Jong K, Nio CY, Hermans JJ et al. High prevalence of pancreatic cysts detected by screening magnetic resonance imaging examinations. Clin Gastroenterol Hepatol 2010; 8: 806-811

[3] Canto MI, Hruban RH, Fishman EK et al. Frequent detection of pancreatic lesions in asymptomatic high-risk individuals. Gastroenterology 2012; 142: 796 -804; quiz e14-e 15

[4] Vege SS, Ziring B, Jain R et al. American gastroenterological association institute guideline on the diagnosis and management of asymptomatic neoplastic pancreatic cysts. Gastroenterology 2015; 148 : 819-822; quiz e12-e13
[5] Scheiman JM, Hwang JH, Moayyedi P. American gastroenterological association technical review on the diagnosis and management of asymptomatic neoplastic pancreatic cysts. Gastroenterology 2015; 148: $824-848$, e22

[6] Lee LS. Diagnostic approach to pancreatic cysts. Curr Opin Gastroenterol 2014; 30: $511-517$

[7] Kadiyala V, Lee LS. Endosonography in the diagnosis and management of pancreatic cysts. World J Gastrointest Endosc 2015; 7: 213 223

[8] Kaimakliotis P, Riff B, Pourmand K et al. Sendai and Fukuoka Consensus Guidelines Identify Advanced Neoplasia in Patients With Suspected Mucinous Cystic Neoplasms of the Pancreas. Clin Gastroenterol Hepatol 2015; 13: $1808-1815$

[9] Lee LS, Wu BU, Banks PA et al. Utility of commercial DNA analysis in detecting malignancy within pancreatic cysts. JOP 2014; 15: 182 188

[10] Jang JY, Park T, Lee $S$ et al. Validation of international consensus guidelines for the resection of branch duct-type intraductal papillary mucinous neoplasms. Br J Surg 2014; 101: 686-692

[11] Sahora K, Mino-Kenudson M, Brugge W et al. Branch duct intraductal papillary mucinous neoplasms: does cyst size change the tip of the scale? A critical analysis of the revised international consensus guidelines in a large single-institutional series Ann Surg 2013; 258: $466-475$

[12] Fritz S, Hackert T, Hinz U et al. Role of serum carbohydrate antigen 19-9 and carcinoembryonic antigen in distinguishing between benign and invasive intraductal papillary mucinous neoplasm of the pancreas. Br J Surg 2011; 98: $104-110$

[13] Shimizu Y, Yamaue H, Maguchi $\mathrm{H}$ et al. Predictors of malignancy in intraductal papillary mucinous neoplasm of the pancreas: analysis of 310 pancreatic resection patients at multiple high-volume centers. Pancreas 2013; 42: $883-888$

[14] Correa-Gallego C, Do R, Lafemina J et al. Predicting dysplasia and invasive carcinoma in intraductal papillary mucinous neoplasms of the pancreas: development of a preoperative nomogram. Ann Surg Oncol 2013; 20: $4348-4355$

[15] Tanaka M, Fernandez-del Castillo C, Adsay V et al. International consensus guidelines 2012 for the management of IPMN and MCN of the pancreas. Pancreatology 2012; 12: 183-197

[16] Springer S, Wang Y, Dal Molin M et al. A Combination of Molecular Markers and Clinical Features Improve the Classification of Pancreatic Cysts. Gastroenterology 2015; 149: 1501-1510

[17] Tanaka M, Chari S, Adsay V et al. International consensus guidelines for management of intraductal papillary mucinous neoplasms and mucinous cystic neoplasms of the pancreas. Pancreatology 2006; 6: $17-32$

[18] Sawhney MS, Al-Bashir S, Cury MS et al. International consensus guidelines for surgical resection of mucinous neoplasms cannot be applied to all cystic lesions of the pancreas. Clin Gastroenterol Hepatol 2009; 7: $1373-1376$

[19] Singhi AD, Zeh HJ, Brand RE et al. American Gastroenterological Association guidelines are inaccurate in detecting pancreatic cysts with advanced neoplasia: a clinicopathologic study of 225 patients with supporting molecular data. Gastrointest Endosc 2016; 83: 1107 1117 